Michael Trestman

The Cambrian Explosion and the Origins of Embodied Cognition

Indiana University, Cognitive Science

Cognitive Science Program

819 Eigenmann

1900 E. 10th St.

Indiana University

Bloomington, IN 47406-7512

Michael.a.trestman@gmail.com

19128564988

Keywords: Cambrian Explosion; Cognition; Evolution; Evolution of Cognition; Evo-devo; Animal Evolution; 


\title{
The Cambrian Explosion and the Origins of Embodied Cognition
}

\begin{abstract}
Around 540 million years ago there was a sudden, dramatic adaptive radiation known as the Cambrian Explosion. This event marked the origin of almost all of the phyla (major lineages characterized by fundamental body plans) of animals that would ever live on earth, as well the appearance of many notable features such as rigid skeletons and other hard parts, complex jointed appendages, eyes, and brains. This radical evolutionary event has been a major puzzle for evolutionary biologists since Darwin, and while our understanding of it has recently improved with new fossil finds, richer molecular phylogenies, and better grasp of ecological, evolutionary, and developmental processes generally, unanswered questions remain. In this paper I argue that a basic cognitive toolkit for embodied, object-oriented, spatial cognition (what I call Basic Cognitive Embodiment) is a practical necessity for control of a large, mobile, complexly articulated body in space. This hypothesis allows us to relate the complexification of animal bodies to the complexification of perception, cognition and behavior in a way that can help to fill in gaps in our emerging picture of the Cambrian Explosion, as well as shed light on the deep evolutionary origins of the mind.
\end{abstract}

\section{What was the Cambrian explosion?}

It seems that until about 540 mya, all animals on Earth were small, slow, squishy, and stupid-in that they lacked brains (Northcutt 2012). Fauna from the preceding time period, the Ediacarian, reveal a diversity of animal body forms, most of which are completely unlike anything today. All of the Ediacarian animals were relatively simple in that they had few tissue types, lacked rigid skeletal elements, and probably had extremely limited capacities for energetic motion. Most of these animals were sessile filter-feeders with frond-like, tube-like or sac-like bodies, although some (e.g. Bomakellia, Kimbarella, Praecambridium sigillum, and Spriggina) have been interpreted - controversially — as early bilaterians with primitively segmented slug-like or worm-like bodies. Exotic forms of bodily symmetry existed which have no 
counterparts among animals of the last half-billion years, such as the trilateral symmetry of the trilobozoans, or the pentamerous symmetry of Arkarua.

All of these animals are difficult to place with any confidence on phylogenetic trees with living animals, because of the strangeness of their anatomies as well as the sketchy nature of the fossils. Classification remains controversial, with some authors assigning them to extant phyla, others proposing extinct phyla (e.g. proarticulata) within the metazoan crown group, and still others interpreting them as stem ${ }^{1}$ metazoa or even as belonging to a separate kingdom, vendobionta (Seilacher 1992). The only fossils from the Ediacarian that can be unambiguously assigned to a crown group are sponges (Narbonne 2005).

The Chengjiang fauna, dated to only 15 million years later, in the early Cambrian, includes a diverse assemblage of animals among which an astounding array of bodies with complex, jointed appendages (including legs, fins, swimming lobes, antennae and complex feeding apparatuses), rigid skeletons, hard armored plates and spines, and sophisticated image-forming eyes (including both camera eyes and a variety of compound eyes) could be found. Although classification of many animals from this period remains controversial, probable representatives of every extant phylum can be found, except those that cannot be expected to leave fossil traces (Erwin 2011).

The disparity between the Ediacarian and Cambrian faunas is striking, and cries out for explanation. In On the Origin of Species, Darwin noted the sudden appearance of the diverse Cambrian fauna ${ }^{2}$ as a 'grave difficulty' for his theory, and appealed to the possibility that earlier fossils had been obscured by geological processes (Darwin 1859 p. 306-310). However, we now have described fauna from before the lower Cambrian boundary, and the discontinuity cannot be ascribed to incompleteness in the fossil record.

To make things even stranger, estimates of the time of ancestral divergence between extant clades, based on 'molecular clock' methods, tend to point to a much

\footnotetext{
${ }^{1}$ The distinction between the stem and crown of a lineage of animals refers to whether or not a group is more distantly related to living members of the group than any other living members. So, for example, crown birds are all of the living birds, and all extinct birds that are as closely related to a living bird as the latter is to any other living bird. Stem birds like archaeopteryx diverged, by definition, from the lineage leading to living birds before the lineages of living birds diverged from each other.

${ }^{2}$ Ediacarian fauna were not recognized until the mid 1900s (Narbonne 2005).
} 
earlier origin of these groups of animals. Estimated divergence times vary widely ${ }^{3}$, but even conservative estimates point to a divergence of extant lineages well before the Cambrian boundary-i.e. before they appear clearly in the fossil record. The lineages leading to the phyla apparently diverged during the Ediacarian (as argued, e.g. by Peterson et. al. 2008) but were mostly represented by morphologically simple stem groups until the Cambrian. After the lower Cambrian boundary, crown metazoan groups began to radiate and evolve their characteristic modern morphologies to an extent that is recognizable in the fossil record. This remarkable time period-the Cambrian Explosion-marked the discernable appearance of all of the major 'bauplans' - fundamental modes of building a bodily architecture during developmentcharacteristic of animals from then until the present.

Each animal phylum ${ }^{4}$ would continue to evolve and diversify (with a few exceptions ${ }^{5}$ ), but in this initial radiation of form, each of the great lines would irreversibly take on a basic pattern of the development of form, which would both scaffold and constrain the evolutionary possibilities of the future. Deuterostomes, an idiosyncratic group defined by their development of an anus prior to developing a mouth, would soon give rise to the first vertebrates, our own lineage of animals with spines; by 530 MYA, Haikouichthys swam the ancient seas, an inch long fish-like animal with gills and proportionally large eyes, a chordate and perhaps even a crown vertebrate (Shu 2008). Arthropods had already devised their versatile signature developmental leitmotif of duplicating and differentiating body segments; by the middle of the Cambrian a spectacular diversity of arthropods inhabited the seas, including early crustaceans, tribolites, and fantastical creatures unlike anything found today, such as opabinia — which had five eyes and a striated trunk-like proboscis with a grasping claw at the end, and the ferocious anomolocarids, which were the largest known animals of

\footnotetext{
${ }^{3}$ As an extreme example, Blair and Hedges (2005) argue, based on molecular evidence, that the deuterostome lineage originated 900 mya, and that jawed vertebrates had already split from agnathans (the group that includes lampreys and hagfish) some 652 mya, deep in the pre-Cambrian. Such early divergence estimates as well as the methods used to infer them are, however, highly controversial.

${ }^{4}$ Phyla are major monophyletic lineages corresponding more or less to the fundamental bauplans. The exceptions being groups characterized by a common bauplan that are probably paraphyletic, such as porifera, which probably encompasses bilateria, and rotifera, which encompasses acanthocephalan (Collins and Valentine 2001).

${ }^{5}$ This is not entirely true. Incredibly, some phyla are represented by only one or a few species and are extremely simple. Perhaps these remarkable animals have remained largely unchanged since the Cambrian earlier. Examples include Placozoa, Micrognathozoa, Xenoturbella, Cycliophora and Phoronida.
} 
the Cambrian and among Earth's first superpredators (Paterson et. al 2011; Parker 2003).

Of particular interest for my purposes here, the Cambrian Explosion marks the appearance of animals with complex, active bodies (CABs). This is a cluster of related properties including:

$\bigcirc$ articulated and differentiated appendages

- many degrees of freedom of controlled motion

○ distal senses (e.g. 'true' eyes)

○ anatomical capability for active, distal-sense-guided mobility (fins, legs, jet propulsion, etc.)

○ anatomical capability for active object manipulation (e.g. chelipeds, hands, tentacles, mouth-parts with fine-motor control)

Today, these characteristics can be found together in only 3 lineages out of the (approximately) 34 described phyla of animals ${ }^{6}$ : arthropods, chordates, and mollusks (especially and perhaps exclusively cephalopods, e.g. squid, octopi and cuttlefish). Arthropods seem to have been the first lineage to cross this threshold of bodily complexity, at the very dawn of the Cambrian. They achieved a spectacular diversity of complex, active bodily forms during the Cambrian era, with a wide array of different anatomical means of locomotion (swimming, crawling and burrowing), and object manipulation (mouthparts and appendages for complex prey handling). They dominated Cambrian ecosystems as apex predators and occupied niches across the entire spectra of trophic level and spatial scale available to animals. While Cambrian chordates are known (Shu 2008), they remained relatively modest in diversity, disparity and apparent ecological dominance until the Ordovician, when conodonts and vertebrates emerged as ecologically important predators. Cephalopods evolved these characteristics last; though mollusks were numerous and diverse in the Cambrian, they did not reach their apogee of bodily and behavioral complexity until much later, with the radiation of coleoid

\footnotetext{
${ }^{6}$ One extinct group of animals, the nectocarids, represent a fascinating possible fourth origin of CABs. Though some authors place nectocarids within cephalopoda, this is unlikely for many reasons, and nectocaris may well be the only described animal from an otherwise unknown phylum (Kroger, Vinther and Fuchs 2011; Mazurek and Zaton 2011).
} 
cephalopods in the Ordovician, during which these extremely active, large-brained animals (e.g. squids, cuttlefish, octopi) rose to global ecological dominance, coming to occupy nearly every marine habitat, as they do today (Kroger, Vinther and Fuchs 2011).

Phylogenetic and morphological diversification during the Cambrian Explosion were by no means restricted to animals with this sort of large complex body. The Cambrian diversification included the appearance of the fundamental body plans for all of the major groups of animals (phyla) that would live on earth until the current time. Many of these lineages would never evolve CABs, but would diversify in other waysfor example, changes in size and shape, metabolic and ecological specializations, symbioses and parasitisms, chemical, visual or mechanical defenses, complex multi-stage life-cycles, and elaborate reproductive systems.

In sum, the Cambrian Explosion was a rapid simultaneous diversification and complexification of many lineages of animals, which included the first appearance of all of the animals that bear discernable phylogenetic relationships to modern animals. It also marked a dramatic increase in the bodily-and correspondingly, behavioralcomplexity of animals, although the evolution of complex active bodies was restricted to a small handful of phyla.

What explains this phenomenon? Why, at the Cambrian Boundary, did many lineages simultaneously begin to radiate, diversify, increase in morphological complexity, and take on stable morphodevelopmental identities that would persist for hundreds of millions of years, until the present? This is the puzzle of the Cambrian Explosion.

\section{What does it mean to explain the Cambrian Explosion?}

What does it mean to try and explain something like the Cambrian Explosion? This is a fraught and complex issue, largely because the target itself is so complex and multifaceted. What are we trying to explain? The Cambrian Explosion is many things at once: 
- Phenomenologically, from our perspective of paleontologists, it is the appearance of certain kinds of fossils in the record, which is then interpreted to signify various sorts of biological changes ${ }^{7}$.

- It is the increase in average maximum body size of multicellular animals.

- It is the origin of certain features of animal bodies: hard parts (e.g. armored plates and spines); differentiated body segments; complex articulated appendages; perceptual organs; movable skeletal elements; complex internal organ systems. Before the Cambrian boundary, there were no animals with these features (or, at best, very few animals with very rudimentary such features).

- It is the appearance of the phyla - of animals that can be recognized as sharing fundamental developmental processes for generating bodily form, and which can therefore be interpreted confidently in terms of their phylogenetic relationships to later animals. Of particular interest is the fact that no new phyla are known to have originated since this period.

- It is the origin of certain developmental technologies/processes capable of giving rise to these sorts of more complex, differentiated bodies, and which correspond to and characterize the phyla.

- It is a radiation or diversification in two senses:

- An increase in phylogenetic diversity or biodiversity at various taxanomic levels. In particular, the number of phyla and classes increased dramatically from the Ediacarian to the Cambrian (Erwin et al. 2011).

- An increase in 'disparity' or the diversity of animal form. Animal bodies evolved that were more different from one another, filling 'morhpospace' (the mathematical conception of the range of possible bodily forms). This almost surely coincided with an increase in diversity of ecological roles, and the origin of a multi-tiered trophic ecology with herbivores and carnivores.

- It is also a complexification of bodily form-it is an increase in the maximum complexity and average complexity of animal bodies. This is different from an

\footnotetext{
${ }^{7}$ This interpretation has not been without its own controversies. Fortey (2001), for example, argued that the apparent 'explosive' radiation of form is an artifact of the fossil record. However, majority that approaches consensus now exists that the Cambrian Explosion was a real phenomenon.
} 
increase in disparity, either of which could in principle have happened without the other. Complexity is of course a vexed notion, but some of the evolution innovations listed above should count as clear examples. The case I am most interested in, again, is what I call 'complex active bodies', bodies with distal senses and strong, articulated limbs capable of active locomotion and object manipulation. I interpret this as corresponding to an increase in the complexity of behavior, and probably an increase in its average energy output as well.

However we choose to single it out, the Cambrian Explosion was a massively distributed event-distributed over space (the entire biosphere) and time (tens of millions of years, and probably several pulses of mass extinction and adaptive radiation (Shu 2008)). This single event was composed of many events-trillions of individual instances of birth, development, organism-interactions such as competition for resources, predation and sexual reproduction, every aspect of life history, and death. Our conception of the Cambrian Explosion largely concerns high-level statistical trends over this vast collection of spatiotemporally localized events. As highlighted above, there are many aspects of it that we could single out for causal-historical explanation, different high-level properties or trends that it seems to exhibit and which make it unique among other stretches of the history of life on planet Earth.

As is generally true of complex historical events, rather than singling out a single well-defined cause-'The Cause'-for the CE, we should see the project of explaining it in terms of identifying causal dependency relations between different events or patterns of events that constitute or precede it. And as with other temporally extended, complex historical events, we can also explore causal relationships within the Cambrian Explosion (i.e. how parts of it depended on other parts of it as it unfolded over time) as well as between parts of the $\mathrm{CE}$ and events that preceded it. It is worth distinguishing between a few different sorts of broad categories of things we might try to explain by picking out causal relationships, which we can concisely label whether, when, how and who explanations:

\section{Whether?}


Numerous conditions had to be in place for the Cambian Explosion to occur. For example, it is trivial that if environmental conditions, for example temperature, had not remained within the tolerance range of life on earth, the Cambrian Explosion would not have occurred (because everything would have died). Picking out a condition that was necessary for the $\mathrm{CE}$ amounts to identifying a 'whether' cause-since whether the Cambrian Explosion occurred depended on whether this condition occurred. There are many suggestions in the literature for whether causes.

\section{When?}

On what previous events did the timing of the Cambrian Explosion depend? What events prior to the start of the C.E. satisfy the following condition: If $\mathrm{x}$ had happened earlier/later, the C.E. would have started earlier/later.

On what contingencies (either prior or during the C.E. itself) did its duration, i.e. the timing of its end, depend? If we can identify distinct component events within the C.E., what temporal dependencies hold between them?

Two of the most striking properties of the Cambrian Explosion are temporal properties: its suddenness and its uniqueness in the history of life; indeed these are to large extent what define the C.E., and single it out as an event in the history of life. The fact that so many of the phyla suddenly (in only a few tens of millions of years) and simultaneous appear is remarkable, and is what merits the moniker of 'explosion.' At least as important is the fact that is the only such event - a small number of animal phyla may have appeared outside of this temporal window, but never before or after was there such a radical increase in the overall diversity and disparity of animal life. Even following the dramatic mass extinction events at the end of the Permian (which killed off some \%95 of the earth's total biodiversity) or the end of the Cretaceous (which wiped out most of the earth's ecologically dominant megafauna, including the non-avian dinosaurs, pterosaurs, mozasaurs and plesiosaurs) there was not a marked increase in the array of morphological diversity exhibited by animals, the way there was in the Cambrian. Many taxa, such as mammals (cites), birds (cites), and teleost fishes (Friedman 2010) increased in intrinsic diversity and disparity following the end Cretaceous extinction, as they expanded to take on ecological roles that had been filled 
by the previously dominant but now extinct non-avian dinosaurs, pterosaurs, and marine reptiles. For example, mammals took on a variety of novel morphologies as the bats gained winged flight, the whales and pinnipeds took to the sea, and other clades as different as ungulates, carnivores, elephants and primates walked, flapped, swam, crawled and climbed their separate trajectories through morhpospace. However, animals as a whole did not increase in phylogenetic or morphological diversity (disparity) in this transition, as they did during the Cambrian Explosion.

\section{How?}

Why did the Cambrian Explosion occur the way it did? What factors affected, not whether or when the CE happened, but how it happened-i.e. which patterns of radiation and/or complexification manifested?

As discussed above, there are many aspect or features of this phenomenon. During this time period, many notable changes occurred in the composition of the fossil record, which we take to indicate changes in the nature of life on earth. We see the appearance of the recognizable phyla that contain all the animals alive today and a rapid increase in taxanomic diversity (number of distinct taxa-classes, orders, genuses, species - within in these larger taxa) and diversity of form or 'disparity'; we see the appearance of larger animals, animals with hard parts, more complex bodies with larger numbers of tissue types, with hard parts, with complexly articulated skeletons and differentiated appendages, animals with distal sense organs (eyes), animals that were capable of active locomotion and manipulation of objects. How are these various trends related causally? Are they all due to some common cause, or do they somehow cause each other?

It is worth noting here another distinction between kinds of causes. On the one hand we might have causation of one event by another event that occurred before the other-call this 'triggering' causation. But another kind of causation is relevant for explaining temporally extended complex historical processes: causation of one ongoing process by another. We might call this 'fueling' causation, rather than 'triggering' causation. What were the triggers of the Cambrian Explosion, and what fueled it as it proceeded? 


\section{Who?}

We can also seek to identify causes that explain who was involved. Why was there simultaneous radiation in several lineages? Why do we see the particular phylogenetic pattern of radiation that we do? There was diversification of many lineages, but there was the sort of complexification I identified, the evolution of complex, active bodies, in only a small subset - two out of the roughly thirty-four phyla that emerged either leading up to, or during, the Cambrian. Why did complex active bodies evolve in the lineages they did and not others? Even now are there only 3 (extant) lineages that have evolved the above suite of characteristics-what was special about them that allowed them to make this transition?

Moreover, we might ask whether the evolution of complex active bodies in arthropods (which seems to have occurred first, very early in, or leading up to, the dawn of the Cambrian), or, later, chordates and cephalopods, had a causal role in driving the wider evolutionary radiation across many lineages that made up the Cambrian Explosion. Arthropods were the largest, most ecologically dominant, most morphologically diverse and most complex-bodied animals in the Cambrian. Did their radiation somehow drive radiation in the other lineages? Did arthropods have a privileged causal role in fueling the Cambrian Explosion?

\section{Explanatory factors proposed by other authors}

In this section I'll review a number of explanatory hypotheses that authors have advanced for various aspects of the C.E. I'll highlight what these explanations can and can’t explain, which will help set the stage for my own proposal.

\section{Environmental explanations}

A variety of changes in the abiotic environment have been identified, which may have been crucial in provided conditions conducive to the Cambrian Explosion. The levels of available oxygen in the air and sea apparently increased markedly towards the 
end of the Ediacarian (Fike and Grotzinger 2006). Higher levels of oxygen made it possible for animal bodies to grow larger, their metabolic, developmental and behavioral processes to be more energetically ambitious. As new, energetically expensive forms of behavior (e.g. locomotion and prey handling), probably played important roles in the Cambrian Explosion (see below), the increase in oxygen levels was probably a necessary (but not sufficient) condition for the Cambrian Explosion. If so, it was certainly a 'whether' cause, since whether or not the C.E. happened depended on whether or not the $\mathrm{O} 2$ levels increased. Some authors (cite) have suggested it may have also been a 'when' cause, i.e. the timing of the Cambrian Explosion may have depended on the timing of this environmental change. However, there was a lag of some ${ }^{*} * *$ million years between the apparent rise of $\mathrm{O} 2$ levels and the appearance of the Cambrian fauna, which makes it doubtful that it specifically determined the timing.

Other authors have suggested that a warming of the globe, ending a lengthy period of extreme glaciations - referred to as 'Snowball Earth', and from which the very name of the Creogenian geologic era is derived-may have been crucial in triggering the Cambrian Explosion (Kirschvink 1992). As with the increase in free oxygen, the warming of the globe was probably a practical requirement (if not a strictly necessary condition) for the Cambrian Explosion, but, similarly, a lag of many millions of years between the end of the last pre-Cambrian glaciation event and the onset of the Cambrian Explosion casts doubt on the importance of the event as a triggering cause.

While large-scale changes in the abiotic environment may be important in understanding how conditions on earth became conducive to complex multicellular animal life, and perhaps helpful in understanding the timing of the Cambrian explosion, there are limits on how much such factors can explain. They may bear on the 'how' of the C.E., in that, if complexification and increase in metabolic ambition and body size were key in also explaining the phylogenetic and morphological diversification (which is plausible), then, in a sense, the increased oxygenation of the environment both triggered and fueled the Cambrian Explosion. However, the details of how this complexification and diversification unfolded are more intimately rooted in the biology of the animals involved, so we must delve into the evolutionary-developmental and ecological details in order to gain a fuller understanding. 


\section{Genetic/Developmental explanations}

An important class of explanations focuses on evolutionary innovations in the genetic control of development. Many of the elements of the genetic regulatory networks that control spatial patterning and tissue differentiation in animal development are highly conserved among all bilateria. This makes it natural to suppose that some of these may have fueled the Cambrian Explosion, by making possible the increases in complexity and diversity. Clearly, the genetic capacity to control the development of morphologically complex bodies was a precondition for complexification, so in that sense, the bilaterian toolkit of genetic regulatory networks was a 'whether' cause of the Cambrian Explosion, as well as a 'how' cause (in that it bears on complexification).

The origin and assembly of genetic regulatory networks may also help to explain one temporal property of Cambrian Explosion: it's uniqueness in the history of life. Early on, these regulatory networks offered a vast unconstrained potential for differently structured modes of development. Over evolutionary time, which, ex hypothesi corresponded to the Cambrian Explosion, this potential became realized as networks of gene regulation evolved to produce more complex developmental processes yielding more complex animal bodies. Genetically, this largely involved the addition of noncoding micro-RNAs which regulate the translation of messenger-RNAs, which actually code for proteins; though most of the toolkit of messenger-RNA is shared among all animals, including all bilaterians as well as the morphologically simpler cnidarians, increase and (conversely) secondarily loss in micro-RNA complexity are correlated with increase and (conversely) loss in morphological complexity, across all animals (Erwin et al. 2011).

In this process, the very genetic-regulatory specificity that produced this morophological complexity constrained the changes in development (and hence morphology) that were later available, through evolution, to a given lineage. Features of development became locked in or 'generatively entrenched' (Wimsatt 1986). Each of the animal phyla are characterized by distinctive, fundamental modes of development; once established, these allowed for the stable development of complex animal bodies, but thereafter they could not be altered at a fundamental level, as any change to the genetic 
regulatory networks that control development at a fundamental level (especially processes early in development) would be catastrophically disruptive to development, and lead to an ontogenetic — and therefore evolutionary — dead end.

So the origin of developmental gene regulatory networks may help to explain the uniqueness of the Cambrian Explosion, as well as, in part, how the complexification of animal bodies was possible at all, but can it explain the timing of its onset? Here things are more problematic. Many of the core elements of genetic regulatory networks are conserved across all bilaterian animals, but these lineages apparently diverged well before the Cambrian Explosion began.

As in the case of the environmental conditions (sufficient $\mathrm{O} 2$ levels, conducive temperature), all of the essential background conditions seem to have been in place at least tens of millions of years before things really took off. Estimates of divergence times between the major bilaterian clades (e.g. deuterostome, ecdysozoa and lophotrocozoa) vary rather widely, but, as noted earlier, even conservative estimates (e.g. Erwin et al. 2011) place this tens of millions of years prior to the start of the Cambrian Explosion. For tens of millions of years, the lineages that would lead to the crown phyla gave way to little phylogenetic or morphological diversification, and to little qualitative complexification. In particular, no animals with complex active bodies were around. Then, quite suddenly, there was an explosive radiation of phylogenetic and morphological diversity in many phyla simultaneously, and a radical increase in complexity in multiple phyla, including the evolution of complex active bodies in at least two taxa (chordates and arthropods).

What explains this lag? Was there some more specific trigger that was needed to set off the 'explosion', even after all of the materiel was in place?

As well, it is unclear how this sort of genetic/developmental explanation might bear on the problem of explaining the 'who' of the Cambrian Explosion. As previously noted, much of the system of developmental gene regulatory networks is shared among all bilaterian phyla. Why then are there such marked differences between phyla in the degree of bodily complexification that unfolded during the Cambrian and afterwards? Why did only three phyla (excluding nectocarids as a possible fourth) evolve complex active bodies with large brains and articulated skeletal-muscle systems? This degree of bodily complexification correlates somewhat (although imperfectly) between phyla with 
increase in complexity of the micro-RNA toolkit (Erwin et al. 2011), but is the genetic change cause or effect at the evolutionary level?

\section{Ecological explanations}

It is likely that the Cambrian Explosion corresponded to a dramatic increase in richness and complexity of the ocean's trophic ecology, i.e. to an increase in the number of levels in the Eltonian pyramid and to the interconnectedness of food webs. The primitive state of bilateria was probably benthic, and either detritivorous or filter-

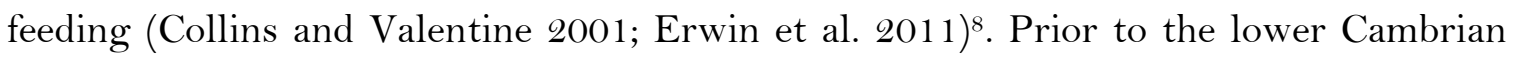
boundary, animals in various bilaterian lineages left the benthos and evolved planktonic forms, thereby gaining access to the rich food source offered by phytoplankton. Butterfield (1997) argues that this may have been crucial in triggering the Cambrian Explosion by dramatically increasing the quantity of biomass available to animals. Erwin et al. (2011) also suggest that the ecosystems engineering activities of animals may have helped to drive the Cambrian Explotion by increasing the biomass available to animals in a couple of ways: a proliferation of filter-feeding sponges late in the Ediacarian may have transferred a large amount of organic matter from the water column to the sediment, and the advent of vertical burrowing by bilaterians early in the Cambrian may have further increased the amount of food available in the sediment by increasing its oxygenation. All or any of these processes would have relaxed food availability as a constraint on metazoan lifestyles, allowing animal lineages to increase in size and metabolic activity. Though this does not imply complexification, it allows it. Like the global increase in available oxygen, and the thawing of 'snowball earth', this helped to provide conditions under which subsequent complexification was possible for animal life.

As bilateria evolved that were big and active enough to prey on other animals, this likely drove a runaway arms race between the predators and their prey, leading to selection for more sophisticated means of catching and killing, as well as better defenses

\footnotetext{
${ }^{8}$ Cnidarians were probably predators much earlier, as evidenced by the commonality of the cnidocyte (the stinging cell used to kill prey) to all crown cnidaria, which diverged around 680 MYA (Erwin et al. 2011),
} 
(Conway Morris 2000). Charles Marshall (2006) argues, based on theoretical arguments and computer simulations by Karl Niklas $(1994,1997,2004)$ that the roughness of a fitness landscape, sensu Wright (1932), is at least partly determined by the number of distinct needs an organism must satisfy in order to survive and reproduce. The rougher the fitness landscape, the more local 'peaks' there are, i.e. the more different ways there are for a lineage to evolve and still be relatively fit. Over evolutionary timescales, roughening of fitness landscapes can therefore drive increases in both diversity and disparity as lineages are driven through morphospace toward local fitness peaks. Marshall argues that by increasing the number of needs that animals had to satisfy in order to survive in a more hostile environment, the advent of predation roughened the fitness landscape for animals in general, and hence drove the increases in diversity and disparity as various lineages evolved different means of exploiting new food sources and protecting themselves from the changing array of predatory threats.

This narrative offers considerable explanatory power, much of which is nonredundant with what is offered by abiotic environmental and genetic/developmental explanations. A widespread predatory arms race could help to explain why the Cambrian Explosion was phylogenetically widespread (i.e., spanning many lineages). It can also help to explain aspects of 'how' animals evolved, in that animals in many different lineages quickly evolved a variety of mechanical defenses (e.g. spines and armor plates), mechanically sophisticated apparatuses for killing and feeding, and more sophisticated means for rapid locomotion.

No doubt ecological considerations (along with the abiotic environmental and genetic/developmental conditions that had to be in place) will play a role in any adequate explanatory narrative about the Cambrian Explosion, but questions remain. In particular, if predatory arms races were important, as many authors have claimed, what kicked off the predatory arms races at the beginning of the Cambrian Explosion? What could explain a qualitative shift in the complexity of the trophic ecology in a way that could drive a roughening of the fitness landscape?

Apparently, the basic essential conditions, both in terms of the abiotic environment (e.g. temperature and 02 ), and the developmental gene-regulatory network technology available to bilaterians, had been in place for some time. Was there some specific innovation in a particular lineage (e.g. the dominant and diverse Arthropods) 
that allowed them to assume the role of predator in such a way as to escalate predatorprey competition to qualitatively new heights, cascading across global ecosystems and driving explosive diversification in many lineages at once?

\section{Specific innovation explanations}

What specific evolutionary innovations, i.e. novel traits, could have had the power to so significantly alter the ecology of the ancient oceans that they could have driven the Cambrian Explosion?

\section{Vision/Eyes}

Andrew Parker has argued forcefully (Parker 2003) that the origin of imageforming eyes was a powerful trigger of the Cambrian Explosion. On his view, the origin of true eyes in early arthropods ${ }^{9}$ (other animals at that point had proto-eyes, capable of detecting light intensity, direction and gradient, but not forming images) allowed this group to rapidly rise to ecological dominance and to exert a powerful and novel array of selective forces on other organisms in virtue of this new ability. For example, other animals upon which visually guided arthropods preyed were now selected for visual crypsis (e.g. camouflage) in order to avoid predator encounters. Prospective prey would have also been more heavily selected for additional mechanical and/or chemical defenses because of the increased probability per time unit of encountering a predator, since predators could now be guided by vision, rather than passively wandering or following chemical gradients. In addition, prey animals that employed chemical defenses would now be selected for warning coloration, which might also select for Batesian mimicry on

\footnotetext{
${ }^{9}$ Parker's views on arthropod phylogeny are heterodox, or at best, controversial. He claims that all crown arthropods, as well as extinct groups such as anomalocaris and opabinia, are derived from trilobites (Parker 2003, p. 217). This is contrary to most recent reconstructions, which consider trilobites to be crown arthropods (Rota-Stabelli, Omar, et al. 2011), and consider anomalacaris, opabinia, and other Cambrian 'oddballs' with well developed eyes to be stem arthropods (Budd and Telford 2009; Giribet and Edgecombe 2012; Paterson, John R., et al 2011). This does not undermine the basic thrust of his argument for the importance of arthropod eyes in driving the C.E., although it does problematize aspects of the narrative he articulates (Parker 2003).
} 
non-toxic animals that looked roughly similar. Other types of visual anti-predation mechanisms could also be employed, such as false eye-spots, motion dazzle.

On top of all of this, any lineage employing visual mechanisms, either by relying on vision, or using visual anti-predatory defenses, might be especially prone to sympatric speciation, as the selective filtering of light frequencies by water creates zones of depth at which different color palettes are present in the environment-so, for example, a coloration that is cryptic at a depth of 300 meters might not be cryptic at a depth of 30 meters, and a coloration that is cryptic at 30 meters might not be cryptic at 3 meters. (Parker 2003, p 103-104). Arthropods themselves, having eyes, could now also be subject to sexual selection for visual features such as the courtship displays of seedshrimp which employ both iridescence and chemical bioluminescence (Parker 2003, ch 5). Sexual selection can be a potent driver of speciation, especially alongside other mechanisms (Ritchie 2007).

All of these aspects of selection for visual properties create additional phenotypic constraints on the 'fitness landscape' for an evolving lineage, which may cause increased diversity and disparity over time, as discussed earlier (Marshall 2006).

I think Parker's insight is important, and makes a non-redundant contribution to our understanding of the Cambrian Explosion, but it leaves open questions.

Parker waffles between identifying the cause as the origin of vision and the origin of eyes, which he distinguishes from proto-eyes by saying that they form images, but the difference is important. As Parker himself highlights, it was visually guided, and hence active predatory behavior-which Parker characterizes as 'search and destroy' predation, rather than ambush predation, or random movement through the environment-by the early arthropods that plausibly drove the predator-prey arms race that cascaded through the world's ecosystems, driving the Cambrian Explosion. But having image forming eyes is not sufficient for active predatory behavior: graft an eye onto a sponge, a placozoan or a houseplant, and it will not become an active predator. Less hypothetically, some lineages have image forming eyes but not active predation, such as ark clams, fan worms, and some gastropod mollusks (Parker 2003 p. 212). Moreover, while Parker seems to think that having eyes also drove the increase in morphological disparity and complexity in arthropods, many lineages of animals have image forming 
eyes and are active predators, such as alciopid bristle worms, box jellyfish, and velvet worms (Parker 2003, p 212), but never achieved morphological disparity or complexity remotely comparable to that of the arthropods. So something is clearly missing.

Vision, or visually guided predation, is part of the story, over and above just the anatomical feature of an image-forming eye; but what is vision? Parker defines it as "the formation of an image or picture from light waves" (Parker 2003 p. 188), but this cannot be right, for the above reasons. An image is formed by an eye grafted onto a sponge, as is one by a camera, but neither the sponge nor the camera can see. In no case can the formation of images per se go very far in explaining the core features of the Cambrian Explosion.

For a conception of vision that bears on the problem at hand, I propose: pick up of information about the environment from patterns in ambient light arrays, and use of that information for control of behavior. Vision in the relevant sense is largely a matter not of the eye per se, but of the brain's ability to extract information from patterns in light, and to make use of that information for controlling behavior in more sophisticated ways. If this is right, than an important question is, what sort of information was most important in driving the Cambrian Explosion? What information must the brain be able to extract from the patterns of light made available by the eye, track over time, integrate or synthesize, and bring to bear on the control of behavior, in ways that can qualitatively change the dynamics of predator-prey relationships sufficiently to drive a cascade of adaptive radiation?

Parker claims that, with the origin of the eye, the biological world suddenly became full of color, and identifies some ways in which color information per se was important for applying novel selection pressures on animals, such as crypsis, warning colorations, mimicry, and sexual selection (Parker 2003, Ch 9). However, this assumption is problematic, as color vision depends on having several different kinds of photoreceptors with different peak response frequencies. Cones and pigments do not fossilize, so it is impossible to know whether Cambrian arthropods had color vision. Moreover, much of the information delivered by vision that is useful for controlling behavior does not require color at all.

What may have been most important for the Cambrian Explosion was the spatial information that animals could suddenly see. Just like color, space had always been 
there. Animals, other living things, and features of the abiotic environment had always had spatial properties such as position, size, distance from each other, occlusion (in between-ness), trajectories, etc., but these properties had existed unknown, in the 'darkness' of having no one to understand them, detect them or track them.

The Cambrian Explosion marks the appearance of animals that could perceive, cognize, and move intelligently in space. The evolution of more sophisticated eyes was part of this important shift, but only part-mostly what had to evolve was a suitably equipped brain. This was largely a cognitive change (in a broad sense of the word 'cognitive'). ${ }^{10}$ All of a sudden, the living world became flooded not just with color, but with the information about space that light can carry. Parker tacitly acknowledges this; as he says, "Importantly (an animal with vision) could easily identify the other animals sharing its environment. It could determine how far away they were, where they were heading, and how fast they were moving. (Parker 2003 p. 273, emphasis added.) However, while acknowledging the importance of spatial properties such as distance, heading and speed, Parker does not pursue the consequences of this line of reasoning.

Vision is not the only sense that carries spatial information. Olfaction is capable of giving information about direction, and even in very simple animals and single-celled organisms, can drive chemotaxis, which involves orientation in space toward the source of the detected chemical, via very simple reflex dynamics and without necessitating a cognitive capacity to pick up or track spatial properties (see Braitenberg, 1986, Chapters 3 and 4$)$.

But a far richer palette of spatial properties is carried by light, and in real-timelight moves at the speed of light (or almost at the hypothetical speed of light, when moving through a medium, but the difference is negligible compared to the processing speed of organic brains), and this means that visual arrays change as quickly as the layout of objects in a scene. This is obviously crucial in the context of agonistic behavioral interactions such as those between predator and prey. Audition, and in

\footnotetext{
${ }^{10}$ Parker is dismissive of the importance of brain evolution in the Cambrian Explosion: "For an eye to work, sizeable brain and nerve cables are required, and these were in part borrowed from other senses. This is the most conceivable way in which an eye can suddenly achieve vision, after its leap from simple progenitors, the light perceivers. What does this borrowing tell us? It indicates that at least some senses had evolved to a reasonable degree of sophistication before the Cambrian, so that they had established a nerve network including brain space. In turn this means they could not have triggered the Cambrian explosion." Parker 2003 p 285
} 
particular echolocation can also be spatially rich (even more so in some cases), but for whatever reason, no animals seem to have developed echolocation before vision. Echolocation has in every case been a secondary adaptation by an animal already keen at spatial cognition to situations where the spatial information carried by light can be greatly supplemented by that carried by sound (e.g. night time for bats, underwater for whales, blindness for some humans (Schenkman and Nillson 2010).

But beyond the ability to detect patterns of energy, such light or sound, from which spatial information can be extracted, the ability to use this information to control behavior requires the ability to integrate information from multiple exteroceptive modalities with proprioceptive information about the spatial relations of parts of the animal's own body to each other and feature of the environment. It also requires the ability to predict the sensory changes caused by the body's own powered movements, in order to cancel them out, delivering a stable, meaningful perception of the spatial layout of the environment (Merker 2005). For these reasons, the capacity to perceive space and use spatial information to control behavior is largely cognitive; the evolution of this capacity required not just the appearance of image-forming eyes, but suitable brains.

\section{Associative learning and neurohormonal stress}

Ginsburg and Jablonka (2010) argue that the origin of the capacity for associative learning may have been a cause of the Cambrian Explosion. Associative learning enhances adaptability during the lifetime of an animal, allowing it to exploit new resources. They claim that this could have played a key role in driving the predatory arms races that fueled the adaptive radiations across the animal phyla. Further, they argue that associative learning can drive increases in morphological and phylogenetic diversity through genetic assimilation (a.k.a. the Baldwin effect). This effect occurs when some novel behavior is consistently learned by animals in a population when exposed to some new environmental stimulus. Over time, the acquisition of this behavior becomes developmentally canalized, which allows it to be acquired faster and more reliable until it ultimately becomes 'innate' or 'instinctive', requiring very little exposure to the environmental stimulus before being expressed (Baldwin 1896; Waddington 1957; West-Eberhard 2003; Bateson 2005). This can drive 
speciation, when different populations of the same species are consistently exposed to different learning environments (Jablonka and Ginsburg 2010; Hardy 1965; Wyles 1983; West-Eberhard 2003). Further, this sort of stabilized behavioral plasticity can have important long-term ecological and evolutionary effects through nicheconstruction (Avital and Jablonka 2000).

Ginsburg and Jablonka argue that as oxygen levels increased through the Ediacarian, animals increased in size, longevity and activity level. The increases in size and activity level drove a centralization of the nervous system, in order to allow integration of sensory inputs and coordination of motor outputs between regions of the body (2010 p. 14), even as the increase in longevity put a premium on learning from past experience, as the probability of situations recurring during a lifetime increased (2010, p. 15). Associative learning emerged simultaneously in many lineages (due to shared features of developmental gene-regulatory networks and neural net architecture) as the constraints on body size, metabolism and longevity due to oxygen availability were relaxed. This opened the door for adaptive behavior in the realms of habitat selection, niche construction, predation and anti-predation, driving the Cambrian Explosion.

Ginsburg and Jablonka also emphasize the potential importance of neurohormonal stress as a factor. Stress is a state of high metabolic activity in response to perceived danger, facilitating adaptive response to that danger (e.g. fight or flight). Although the relationship between stress and associative learning is a bit unclear in their narrative, the two are related, in that stressful situations are powerfully conducive to associative learning, and can facilitate rapid long term learning, for example in cases like fear conditioning or taste aversion. Furthermore, it is plausible that neurohormonal stress evolved early in the Cambrian explosion with the complexification of bodies and nervous systems, as 1) animals first became capable of energetic, coordinated, wholebody behavioral responses to danger, and 2) the importance of predation as a selection increased radically.

Ginsburg and Jablonka argue that early in the Cambrian, phylogenetic diversification may have been extremely rapid because physiological stress had evolved, but physiological stress management had not. They marshal evidence that neurohormonal stress can increase levels of genetic transposition, which can increase 
evolvability (Zeh et al. 2009; Oliver and Greene 2009), as well as by destabilizing the genome through changes in epigenetic marks, "which can lead to altered patterns of mitotic and meiotic pairing, to chromosomal re-patterning, to mutation-prone repair, as well as triggering transposition." (Ginsburg and Jablonka 2010) Extant organisms have systems to ameliorate and suppress the genome and epigenome destabilizing effects of stress, but during the Cambrian Explosion, these systems probably had not evolved. Thus, the evolutionary explosiveness of the Cambrian may have been in part due to the fact that stress had evolved, but stress management had not.

I think Ginsburg and Jalonka offer some important insights here, but I think their line of reasoning can be continued by considering that there are different sorts of associative learning, some more complex than others. This may help to shore up a major weakness of their account, which is that it does not take any special notice of the privileged role arthropods played in the Cambrian Explosion. If associative learning and neurohormonal stress are phylogenetically widespread, why is there such a markedly uneven distribution of complexification, diversification, and ecological dominance throughout the Cambrian Explosion? This issue is especially acute, since Ginsburg and Jablonka's explanation largely hinges on the relevance of associative learning for increased efficacy in complex behaviors, in connection with increased selection for bodily complexity. I suggest that, while associative learning may be widespread, a more specific type of associative learning (and perception and cognition more broadly), objectoriented associative learning, perception and cognition, is restricted to only a handful of taxa. It is a crucial component of the suite of cognitive capacities I call Basic Cognitive Embodiment (see below).

\section{My proposed explanation: Basic Cognitive Embodiment}

I propose that a fundamental cognitive/perceptual toolkit for tracking certain spatial properties and relations, what I'll call Basic Cognitive Embodiment (BCE), is required for effective control of a complex active animal body (CAB). When the first animals evolved BCE, it first allowed the rich, real-time spatial information provided by compound or lens eyes to guide active mobility and object manipulation; this created intense selection pressure for the increased power and articulation of skeletomuscular 
systems, and hence was a driver of morphological diversification and also bodily complexification, in a way that bears directly on the predator-prey arms races widely thought to be important in fueling the Cambrian Explosion. Arthropods seem to have acquired BCE first, which explains their uniquely explosive diversification, active bodily complexification, and rise to dominance of Cambrian ecosystems. The appearance of spatially savvy, visually guided predators with complex active bodies radically changed the array of selection pressures for animals in general, across many phyla, putting a premium on mechanical defenses, crypsis, chemical defense, remote sensing, mobility, selective movement, and choice and construction of safe micro-habitats, driving the widespread adaptive radiation that makes up the Cambrian Explosion. Chordates seem to have acquired BCE not long after — as evidenced by the presence in Cambrian strata of complex, large-eyed, active chordate bodies streamlined for efficient powered swimming (Shu 2008). Their diversification was likely surpressed by the already dominant arthropods, but their presence in the Cambrian fauna represents a disproportionate contribution to overall complexity and disparity of animal bodies. Cambrian chordates such as Haikouichthys were the first animals to take on a 'fish-like' morphology — streamlined, visually guided swimmers powered by undulation of the body along its main axis (Shu et al. 2008), a body morphology that would characterize many vertebrate lineages over the course of history (e.g. placoderms, sharks, teleost fish, several lines of Mesozoic marine reptiles, cetaceans and pinnipeds). They were some of the only animals of their size in the Cambrian to lack obvious mechanical defenses like hard shells or spines, so they probably survived by being able to out-swim larger, less streamlined arthropod predators (as do thousands of species of small fish today).

BCE is a cognitive toolkit for pickup, tracking, and use (for control of behavior) of certain information from the environment. The defining properties of $\mathrm{BCE}$ are that it is 1) spatial, 2) object-oriented, and 3) agentive (or 'action-oriented'):

$\mathrm{BCE}$ is an awareness of spatial properties such as:

- Orientation to body (relative to the body's structural axes and axes of active motion), and also to body's trajectory through space and other action capacities. 
- Distances (relative to action capacities)

- Trajectories, rates of travel, and other spatial propensities

○ Occlusion, concealment (including partial)

BCE is also the ability to perceive and cognize in a way that is object-oriented. Objects, in the relevant sense, are co-tracked clusters of associated properties including:

- Nonspatial properties such as colors, scents, flavors, and pitches

- Affordances (opportunities for behavioral exploitation, relative to bodily capacities and needs)

- Spatial properties - see above; for the purposes of action, it is co-location and spatial co-tracking (at least as much as robust association) that bind a cluster of properties into an object, and also govern almost every aspect of the animals interaction with it.

$\mathrm{BCE}$ is also an agentive or action-oriented bodily self-awareness, i.e. an awareness of the animal's own capacities for moving through space and influencing objects around it. Examples include:

- Judging distances relative to one's own capacity for movement

- Canceling out sensory change caused by one's own movements in order to build stable perception of the world.

- Judging relevance of objects and situations in the environment relative to they body's needs and capacities.

$\mathrm{BCE}$ is an interrelated suite of cognitive capacities, the origin of which represents a kind of qualitative 'complexity threshold' in evolution, because it is required for control of a complex, active body ${ }^{11}$. Without the ability to pick up and track spatial track information about spatial properties and relations, in relation to the body's own axes and ranges of motion and capacities for motion, both distal senses and

\footnotetext{
${ }^{11}$ See Merker (2005) for a discussion of similar considerations of control of complex, active bodies, albeit one with a different focus and very different explanatory aims.
} 
anatomical capacities for powered locomotion and object manipulation would be useless. Further, these capacities can only be adaptively brought to bear if information about the environment is organized into clusters related to salient objects, such as food items, potential predators, competitors and mates, or features of the habitat (e.g. hiding places). In order to be brought to bear on control of behavior, such object-oriented clusters of information must include spatial properties and relations (e.g. distances, sizes, shapes, trajectories, internal structure of hard and soft parts) relative to the action capacities of the animal's body.

The origin of this suite of capacities in a lineage of animals can cause dramatic adaptive radiation in that lineage as well as shape entire ecosystems, as evidenced by the fact that the three extant lineages that possess it-vertebrates, arthropods, and cephalopods-display enormous morphological diversity and disparity compared with the other thirty or so phyla of animals, and have dominated the world's ecosystems from the Cambrian until today. When the brain of the ancestral arthropod first attained BCE, this opened up a vast array of new evolutionary-developmental possibilities for more complex bodies and coherent, complex, intelligent behavior. This put selection pressure on this lineage for variations that realized these possibilities. In particular, this drove up the adaptive value of:

- Body structures facilitating locomotion, such as legs for walking or crawling, and fins, legs, lobes and general musculature for swimming.

- Body structures facilitating manipulation of objects, in particular in the context of food-handling, such as anterior limbs, mouth parts, chelicerae and antennules ${ }^{12}$.

- Fancy distal exteroceptors such as improved eyes, better hearing, etc.

- Improved capacity for memory, i.e. for learned associations between properties of salient objects and contexts.

- Canalization of development of adaptive perception/action patterns.

${ }^{12}$ See Waloszek et al. (2007) for a fascinating review of the early diversification of arthropod head structures, most of which apparently functioned in food handling of one sort or another. The authors claim that this was an important driver of arthropod evolution, in line with my hypothesis. 
This radiation in the arthropods created a cascade of novel selection pressures on other lineages, due largely to the novel capacities of arthropods as complex, active bodied predators. This introduction of strong selection pressure due to diverse, formidable predators restructured the fitness landscape for animals in other lineages as well as arthropods themselves. Predators and prey co-evolved in a kind of run away arms race, driving a phylogenetically widespread process of explosive diversification.

My proposal makes a non-redundant contribution to the landscape of explanations of the Cambrian Explosion, in that it unifies and builds upon the merits of existing proposals, in particular those of Parker (2003) and Ginsburg and Jablonka (2010), as well as the widely shared premise that predator-prey coevolution was important. It bears on explaining aspects of when, how, and who questions about the Cambrian Explosion in the following ways.

When: The first acquisition of BCE by animals was a triggering cause of explosive adaptive radiation as well as complexification and diversification of complex, active morphology, in the relevant lineages (arthropods first). It also caused extensive adaptive radiation in others due to selection pressures. This explanation identifies a particular evolutionary event (the origin of BCE in arthropods) as a triggering cause, an event on which the timing of the onset of the Cambrian Explosion depended. If arthropods had acquired BCE later or earlier, then the Cambrian Explosion would have begun later or earlier. It also identifies internal causal dependence relations, i.e. between the arthropod radiation and complexification, and the wider metazoan radiation.

Who/How: My hypothesis would also explain the phylogenetic patterns of radiation and complexification that we see in the Cambrian Explosion. BCE is difficult to evolve. It does not simply follow along from selection pressure due to predation or ecological competition, but requires some (unkown) set of preadaptations in neural control architecture, which must be to some extent fortuitous - there is no foresight in evolution, so BCE cannot evolve 'in order to allow' bodily complexification. It therefore represents a strong constraint on the evolution of complex, active animal bodies-a lineage of animals cannot evolve CABs without first acquiring BCE. Therefore, the chief constraint determining whether or not a lineage evolves complex active bodies is 
cognitive, rather than essentially genetic ${ }^{13}$ or environmental. Once animals in a lineage have the developmental capacity for brains capable of picking-up spatial information (as outlined above in the description of $\mathrm{BCE}$ ) and bringing it to bear on the control of behavior, this makes a wide range of morphological possibilities available to that lineage. If ecological conditions are ripe, some of these morphological possibilities will be strongly selected for. However, the effects of this 'opening up' of a new array of evolutionary-developmental possibilities are not limited to the taxon that experiences it. As that taxon radiates and the animals that make it up complexify, this exerts strong ecological pressures on the rest of the biosphere.

This hypothesis of constraint helps to explain the pattern of radiation and complexification seen during the Cambrian Explosion (and indeed afterward), which none of the other hypotheses reviewed here can explain: despite phylogenetic and morphological diversification across nearly all of the phyla, complex bodies appear in exactly three extant phyla. In each of the crown groups with complex active bodies (arthropods, chordates, and cephalopod mollusks), BCE, and indeed more sophisticated forms spatial cognition built upon the foundation of $\mathrm{BCE}$ (such as visual place recognition, multimodal path integration, and cognitive maps), are widespread or universal. While other animal lineages possess image-forming eyes, associative learning, and developmental gene-regulatory networks, no lineage possesses CABs without BCE, or BCE without CABs.

The rise in ecological dominance of the arthropods as complex, active bodied predators seems to have been an important factor in driving the Cambrian diversification, not just in their own lineage, but for animals generally. Therefore, the origin of BCE in arthropods should therefore be considered an important causal factor in the Cambrian Explosion, part of the complex weave of causes at the geneticdevelopmental, behavioral and ecological levels that, together, brought forth this unique and truly seminal episode in the history of life on earth.

${ }^{13}$ Although of course a genetic toolkit for brain development is a prerequisite for having a brain. But remember that many lineages share the basic toolkit of developmental generegulatory networks without evolving CABs. 


\section{References}

Baldwin, J.M., 1896. A new factor in evolution. Am. Nat. 30 (441-451), 536-553.

Bateson, P., 2005. The return of the whole organism. J. Biosci. 30, 31-39.

Blair, Jaime E., and S. Blair Hedges. 2005. "Molecular phylogeny and divergence times of deuterostome animals." Molecular Biology and Evolution 22.11: 2275-2284.

Braitenberg, Valentino. 1986. Vehicles: Experiments in synthetic psychology. MIT press.

Butterfield, Nicholas J. 1997. "Plankton ecology and the ProterozoicPhanerozoictransition.” Paleobiology2,3(2) pp. 247-262.

Budd, Graham E. 2003. "The Cambrian fossil record and the origin of the phyla." Integrative and comparative biology 43.1: 157-165.

Budd, Graham E. "Palaeontology: Cambrian nervous wrecks." Nature 490.7419 (2012): 180-181.

Budd, Graham E., and Maximilian J. Telford. 2009. "The origin and evolution of arthropods."

Cabej, R.N., 2008. Epigenetic Principles of Evolution. Albanet Publishing, Dumont, NJ.

Chen, J. Han, et al. "Lower Cambrian vertebrates from south China." Rise of the dragon: readings from Nature on the Chinese fossil record (2001): 175.

Collins, A., and Valentine, J. 2001. Defining Phyla: evolutionary pathways to metazoan body plans. Evolution and Development 3:6, 432-442.

Conway Morris, Simon. The Cambrian “explosion”: Slow-fuse or megatonnage?

Darwin, C. R. 1859. On the origin of species by means of natural selection, or the preservation offavoured races in the struggle for life. London: John Murray. [1st edition]

Erwin, Douglas H., et al. 2011. "The Cambrian conundrum: early divergence and later ecological success in the early history of animals." Science 334.6059: 1091-1097.

Fike, D.A., Grotzinger, J.P., Pratt, L.M., Summons, R.E., 2006. Oxidation of the Ediacaran ocean. Nature 444, 744-747. doi:10.1038/nature05345. 
Friedman, Matt. "Explosive morphological diversification of spiny-finned teleost fishes in the aftermath of the end-Cretaceous extinction." Proceedings of the Royal Society B: Biological Sciences 277.1688 (2010): 1675-1683.

Fortey, R., 2001. The Cambrian explosion exploded? Science 293 438-439.

Giribet, Gonzalo, and Gregory D. Edgecombe. "Reevaluating the arthropod tree of life." Annual Review of Entomology 57 (2012): 167-186.

Halanych, Kenneth M. "The new view of animal phylogeny." Annual Review of Ecology, Evolution, and Systematics (2004): 229-256.

Hardy, A., 1965. The Living Stream: A Restatement of Evolution Theory and its Relation to the Spirit of Man.

Harvey, Thomas HP, Maria I. Vélez, and Nicholas J. Butterfield. 2012. "Exceptionally preserved crustaceans from western Canada reveal a cryptic Cambrian radiation." Proceedings of the National Academy of Sciences 109.5: 1589-1594.

Jones, David, et al. "The sharpest tools in the box? Quantitative analysis of conodont element functional morphology." Proceedings of the Royal Society B: Biological Sciences 279.1739 (2012): 2849-2854.

Kirschvink JL. 1992. Late Proterozoic low-latitude global glaciation: the snowball Earth. In The Proterozoic Biosphere: A Multidisciplinary Study, eds. JW Schopf, C Klein, pp. 51-58. Cambridge, UK: Cambridge Univ. Press. 1348 pp.

Kroger B, Servais T, Zhang Y. 2009. The Origin and Initial Rise of Pelagic Cephalopods in the Ordovician. PLoS ONE 4(9): e7262.

Kroger, B., Vinther, J., and Fuchs, D. 2011. Cephalopod origin and evolution: a congruent picture emerging from fossils, development and molecules. Bioessays 33: $602-613$.

Kroger, B. and Zhang, Y. 2009. Pulsed cephalopod diversication during the Ordovician. Palaeogeography, Palaeoclimatology, Palaeoecology 273: 174-183

Ma, Xiaoya, et al. "Complex brain and optic lobes in an early Cambrian arthropod." Nature 490.7419 (2012): 258-261.

Mazurek, Dawid, and Michal Zaton. 2011 . "Is Nectocaris pteryx a cephalopod?." Lethaia 44.1: 2-4.

Merker, B. (2005) The liabilities of mobility: A selection pressure for the transition to consciousness in animal evolution, Consciousness Eீ Cognition, 14, pp. 89-114. 
Narbonne, Guy M. "THE EDIACARA BIOTA: Neoproterozoic Origin." Annu. Rev. Earth Planet. Sci 33 (2005): 421-42.

Niklas KJ. 1994. Morphological evolution through complex domains of fitness. Proc. Natl. Acad. Sci. USA 91:6772-79

Niklas KJ. 1997. Effects of hypothetical developmental barriers and abrupt environmental changes on adaptive walks in a computer generated domain for early vascular land plants. Paleobiology 23:63-76

Niklas KJ. 2004. Computer models of early land plant evolution. Annu. Rev. Earth Planet. Sci. 32:47-66

Northcutt, R. Glenn. 2012. "Evolution of centralized nervous systems: Two schools of evolutionary thought." Proceedings of the National Academy of Sciences 109. Supplement 1: 10626-10633.

Oliver, K.R., Greene, W.K., 2009. Transposable elements: powerful facilitators of evolution. BioEssays 31, 703-714. doi:10.1002/bies.200800219.

Paterson, John R., et al. "Acute vision in the giant Cambrian predator Anomalocaris and the origin of compound eyes." Nature 480.7376 (2011): 237-240.

Peterson, Kevin J., et al. 2008. "The Ediacaran emergence of bilaterians: congruence between the genetic and the geological fossil records." Philosophical Transactions of the Royal Society B: Biological Sciences 363.1496: 1435-1443.

Rota-Stabelli, Omar, et al. "A congruent solution to arthropod phylogeny: phylogenomics, microRNAs and morphology support monophyletic Mandibulata." Proceedings of the Royal Society B: Biological Sciences 278.1703 (2011): 298-306.

Ritchie, Michael G. "Sexual selection and speciation." Annu. Rev. Ecol. Evol. Syst. 38 (2007): 79-102.

Schenkman, Bo N., and Mats E. Nilsson. "Human echolocation: Blind and sighted persons' ability to detect sounds recorded in the presence of a reflecting object." Perception 39.4 (2010): 483.

Seilacher, A. 1992. "Vendobionta and Psammocorallia: lost constructions of Precambrian evolution". Journal of the Geological Society, London 149 (4):

Shu, D. 2008. "Cambrian explosion: Birth of tree of animals." Gondwana Research $\mathbf{1 4}$ p.219-240

Stanley, Steven M. 1973. “An Ecological Theory for the Sudden Origin of Multicellular 
Life in the Late Precambrian.” Proceedings of the National Academy of Sciences 70 (5): 1486. . 1992. "Can Neurons Explain the Cambrian Explosion?" Geological Society of

America Abstracts with Programs 24:A45.

Stevens, Martin, et al. "Motion dazzle and camouflage as distinct anti-predator defenses." BMC biology 9.1 (2011): 81.

Waloszek, Dieter, et al. "Evolution of cephalic feeding structures and the phylogeny of Arthropoda." Palaeogeography, Palaeoclimatology, Palaeoecology 254.1 (2007): 273-287.

Waloszek, Dieter, Junyuan Chenb, Andreas Maasa, Xiuqiang Wang. 2005. Early Cambrian arthropods - new insights into arthropod head and structural evolution Arthropod Structure \& Development 34: 189-205

West-Eberhard, M.J., 2003. Developmental Plasticity and Evolution. Oxford University Press, Oxford.

Wimsatt WC. 1986. Developmental constraints, generative entrenchment, and the innate-acquired distinction. In Science and Philosophy, Integrating Scientific Disciplines, ed. W Bechtel, pp. 185-208. Dordrecht: Martinus Nijhoff

Wyles, J.S., Kunkel, J.G., Wilson, A.C., 1983. Birds, behavior, and anatomical evolution. Proc. Natl. Acad. Sci. USA 80, 4394-4397.

Zeh, D.W., Zeh, J.A., Ishida, Y., 2009. Transposable elements and an epigenetic basis for punctuated equilibria. BioEssays 31, 715-726. doi:10.1002/bies.200900026. 